\title{
Hydrothermal coupling in a self-affine rough fracture
}

\author{
A. Neuville, ${ }^{*}$ R. Toussaint, and J. Schmittbuhl \\ Institut de Physique du Globe de Strasbourg (IPGS), CNRS, and Université de Strasbourg (EOST), 5 rue Descartes, \\ 67000 Strasbourg, France
}

(Received 31 March 2010; published 21 September 2010)

\begin{abstract}
The influence of the multiscale fracture roughness on the heat exchange when a cold fluid enters a fractured hot solid is studied numerically on the basis of the Stokes equation and in the limit of both hydrolubrication and thermolubrication. The geometrical complexity of the fracture aperture is modeled by small self-affine perturbations added to a uniform aperture field. Thermal and hydraulic properties are characterized via the definition of hydraulic and thermal apertures both at microscopic and macroscopic scales and obtained by comparing the fluxes to the ones of flat fractures. Statistics over a large number of fracture configurations provide an estimate of the average behavior and its variability. We show that the long-range correlations of the fracture roughness induces strong channeling effects that significantly influence the hydraulic and thermal properties. An important parameter is the aspect ratio (length over width) of the fracture: we show, for example, that a downstream elongated rough fracture is more likely to inhibit the hydraulic flow and subsequently to enhance the thermal exchange. Fracture roughness might, in the opposite configuration, favor strong channeling which inhibits heating of the fluid. The thermal behavior is in general shown to be mainly dependent on the hydraulic one, which is expressed through a simple law.
\end{abstract}

DOI: 10.1103/PhysRevE.82.036317

PACS number(s): 47.56. $+\mathrm{r}, 44.05 .+\mathrm{e}, 47.11 . \mathrm{Bc}, 44.30 .+\mathrm{v}$

\section{INTRODUCTION}

Among situations where heat exchange between a passing fluid and a fractured medium is of central importance, geothermics is an intensively developing field. Deep enhanced geothermal systems (EGSs) are based on the energy extraction obtained when a cold fluid is injected from the surface inside a hot fractured massif at depth and extracted after circulation in the open fractures possibly artificially created from an hydraulic or chemical stimulation (e.g., the EGS pilot plant in Soultz-sous-Forêts, France [1-4]).

The efficiency of the heat exchange depends on the balance between conductive and convective heat fluxes. The former is mainly dependent on the geometry of each individual interface, i.e., facing fracture surfaces, but the latter is definitively related to the hydraulic properties of the fracture network, which results from the network connectivity and the fracture permeability.

Hydraulic conductivity of fractured rocks has been intensively studied for different motivations. For instance, the hydraulic properties of the crystalline aquifer of Ploemeur, France have been studied by Le Borgne et al. [5] to address water supply issues. Another example is the mitigation of radionuclide migration which has been forecasted in the case of the repository site for nuclear wastes storage in Äspö, Sweden on the basis of a discrete fracture network [6]. The modeling of the transport properties of fracture networks is actually a very active research area. A classical approach is to model the flow paths via parallel interacting flat fractures [7]. More advanced studies address the effects of the connectivity and correlations of the fractures (e.g., in Refs. [8,9]).

In many models of hydraulic or hydrothermal flow developed so far, the geometry of each fracture of the network is

\footnotetext{
*amelie.neuville@unistra.fr
}

nevertheless considered as simple, e.g., as parallel plates with a simple geometry of the edge as an ellipse or a polyhedron. This is the case in most fracture network models used for geothermal $[10]$ or for fluid transport applications $[8,9]$. The nontrivial character of the fracture aperture geometry is however very likely to influence the fracture flow given their complex real geometry. Most natural fracture surfaces are indeed self-affine objects. Surprisingly, the complexity of the multiscale properties of the fractures has some remarkable simplicity, in the sense that their Hurst exponent is very robustly around 0.8 [11]. Exceptions however exist like for fractures in sandstones where the Hurst exponent is $0.5[12,13]$ or in glassy ceramics which show an exponent close to 0.4 [14].

The aperture between fracture surfaces is subsequently far from flat in particular if facing fracture surfaces are uncorrelated, at least at small scales $[15,16]$. A rough self-affine aperture is indeed defined between two uncorrelated selfaffine fracture surfaces or between two identical self-affine fractures translated tangentially to their average plane by a translation larger than the scale under study. Self-affine apertures have been shown to be responsible for tortuous flow path. The related channeling of the fluid flow was experimentally observed in rough fractures $[17,18]$ and modeled using lubrication approximation with Reynolds equation (e.g., [13,19-22]). The applicability of such an approximation has been studied, e.g., in Refs. [23-25]. Extension of this situation was considered, for example, by Plouraboué et al. [26] where the Reynolds equation is coupled to the chemical convection-diffusion study. More advanced hydraulic simulations including the solving of the Navier-Stokes equation have been proposed either considering simplified geometry $[23,27]$ or more recently within a realistic fracture geometry [28-32]. However, these recent simulations require heavy computations (although different methods are available) and are therefore not fully compatible with statistical 
approaches where a large number of realizations are necessary.

Beyond the problem of mass flow in rough fractures, different kinds of numerical simulations have already been proposed to account for hydrothermal coupling. As a first approach, analytical solutions have been obtained to compute the heat flux along parallel circular cracks embedded in a three-dimensional (3D) infinite medium using simplified heat equations [33]. At large scale and for long-term predictions, models like that of Bataillé et al. [10] have been proposed to predict the response of geothermal reservoirs. This type of finite-element model includes conduction and free and forced convection, but reduces the geometry of the hydraulic network to a double permeability distribution to account for both matrix and fracture transport. A variety of more complex models have also been proposed like the modeling of a 3D network of fractures organized according to geological observations and completed with stochastic fractures for underdetermined parts of the model [34], or that for Soultzsous-Forêts, France by Rachez et al. [35] or that of Kolditz and Clauser [36] for Rosemanowes, UK.

In the present study, we focus on the hydrothermal coupling at the fracture scale where the channeling effect is expected to affect not only the fluid transport properties, but also the heat flux properties, as suggested by Kolditz and Clauser [36] who proposed that the discrepancy between classical heat model predictions and real observations could be due to flow channeling resulting from fracture roughness. We aim at obtaining from the microscopic analysis of the flow at scales of the fracture asperities the macroscopic parameters (i.e., the hydraulic transmissivity and the characteristic thermalization length) that govern the efficiency of the fluid mass and heat transport through the overall fracture. This will allow us to coarse grain the description of the effect of microscopic asperities, i.e., the fracture roughness, on the hydraulic and thermal behavior in large-scale network models as the ones mentioned above. The upscaling from the microscopic asperity scale to the fracture scale is done via a systematic statistical analysis of the macroscopic flow parameters, for a large set of stochastic synthetic fracture surfaces, described with a few key parameters for such apertures: average aperture and standard deviation. The macroscopic parameters obtained after the upscaling reduce to two: the hydraulic transmissivity, characterizing the fluid mass transport, and the other one characterizing the efficiency of the heat exchange between the rock and the fluid. This exchange is expressed via the characteristic length $R$ in a macroscopic law of type

$$
(\boldsymbol{q} /\|\boldsymbol{q}\|) \cdot \nabla_{2} \bar{T}-\left(\bar{T}-T_{r}\right) / R=0
$$

with $\bar{T}$ as a fluid temperature, $T_{r}$ as the temperature in the surrounding rock, $\boldsymbol{q}$ as the fluid flux integrated over the fracture thickness, and $\nabla_{2}$ as the two-dimensional (2D) gradient operator along the fracture plane. The hydrothermal modeling is performed as in [3]. The present study is in the framework of the lubrication approximation [37] which implies that the Reynolds number is small and that the fracture walls are locally flat enough to provide a mainly in-plane velocity field, with a negligible component normal to the mean fracture plane. We propose to extend the lubrication approximation to the thermal fluxes. By balancing heat conduction and forced convection we obtain a three-dimensional temperature law which will then be reduced to a $2 \mathrm{D}$ temperature equation by averaging it along the thickness of the fracture as proposed, e.g., by Turcotte and Schubert [38].

Section II describes our geometrical model of the fracture aperture based on a self-affine scaling invariance. In Sec. III, using lubrication approximations, we obtain the bidimensional pressure and thermal equations when a cold fluid is injected through a fracture in a stationary regime. As a first step, the temperature within the surrounding rock is supposed to be hot and constant (in time and space), and the density of the fluid is considered as constant. We show that at a coarse-grained scale, the $2 \mathrm{D}$ equation for heat flux is identical to the one for parallel plates [Eq. (1)], but with a characteristic thermalization length associated with an aperture (named thermal aperture), different from the geometrical aperture (also often labeled as the mechanical aperture). Other relevant quantities are defined to describe the hydraulic and thermal behaviors at local and macroscopic scales. The numerical approach is described in detail in Sec. IV. Equations are discretized using a finite difference scheme and solved with a biconjugate gradient method. The numerical hydraulic and thermal results are, respectively, set out in Secs. IV and $\mathrm{V}$. In each of these sections, we first describe the results for a given fracture morphology (locally and macroscopically), then averaged trends of macroscopic parameters that are observed statistically from large sets of synthetic fractures.

\section{DESCRIPTION OF THE ROUGHNESS OF THE FRACTURE APERTURE}

The roughness of a self-affine surface is statistically invariant upon an isotropic scaling within its mean plane $(x, y)$, while on the perpendicular direction $z$ the scaling is anisotropic. Indeed, it is statistically invariant under the scaling transformation $x \rightarrow \lambda x, y \rightarrow \lambda y, \Delta z \rightarrow \lambda^{\zeta} z$ [39-41], where $\zeta$ is called roughness exponent or Hurst exponent. A self-affine geometrical model has been experimentally shown to be a realistic description of natural rock surfaces $[11,16,42,43]$, with Hurst exponent equal at large scale to $\zeta \simeq 0.8$ for many kinds of natural fractures and material surfaces [42-45] and $\zeta \simeq 0.5$ for sandstones $[46,47]$. It is important to note that a self-affine surface having a roughness exponent smaller than 1 is asymptotically flat at large scales [48]. Accordingly, a self-affine topography can be seen as a perturbation of a flat interface. On the other end of the scales, the local slope of a self-affine surface diverges at small scales, and the maximum slope of such surface is determined by the lower cutoff of the self-affine behavior-corresponding, e.g., to granular diameter when present. In principle, modeling a flow boundary condition along such surface requires us to check that the macroscopically obtained result does not depend on such lower cutoff.

The aperture is the space between the facing fracture surfaces. Our study is limited to the case where two noncorrelated fracture surfaces with the same roughness exponents 
are facing each other. Subsequently the aperture $a(x, y)$ is also a self-affine function with the same Hurst exponent which fulfills the following property $[39,40]$ :

$$
\lambda^{\zeta} \operatorname{Prob}\left(\lambda^{\zeta} \Delta a,[\lambda \Delta x, \lambda \Delta y]\right)=\operatorname{Prob}(\Delta a,[\Delta x, \Delta y]),
$$

where $\operatorname{Prob}(\Delta a,[\Delta x, \Delta y])$ is the probability to get an aperture difference $\Delta a$ between two points separated by the distance $[\Delta x, \Delta y], \lambda$ is an arbitrary scaling factor, and $\zeta$ is the roughness exponent.

The self-affine aperture field is numerically obtained by first generating a white noise $\epsilon(x, y)$ [49] on a grid of size $2 n_{x} \times 2 n_{y}$ with a square mesh size $d$. Then the statistical spatial correlations are introduced by multiplying the 2D Fourier transform of the white noise $\widetilde{\boldsymbol{\epsilon}}\left(k_{x}, k_{y}\right)$ by $\|\boldsymbol{k}\|^{(-1-\zeta)}$ [50], where $\boldsymbol{k}$ is the wave vector. When desired, a lower cutoff length scale $l_{c}$ can be introduced by filtering as follows: if $\|\boldsymbol{k}\|$ $\geq \pi / l_{c}, \tilde{a}\left(k_{x}, k_{y}\right)=0$. Finally, we perform the inverse Fourier transform of $\tilde{a}\left(k_{x}, k_{y}\right)$ and normalize it to get a synthetic aperture $a(x, y)$ with an average $A$ and a root mean square (RMS) $\sigma$. Using different seeds of the random generator of the white noise, it is possible to generate independent selfaffine aperture morphologies showing different patterns, even if they share the same roughness exponent chosen equal to $\zeta=0.8$, the same mean aperture $A$, and the same RMS $\sigma$. The upper limit of $\sigma$ is provided by the condition of positive aperture, i.e., we prevent contact between the fracture faces to keep a constant simple boundary geometry of the domain where the equations are solved. In practice $a$ is imposed to range between $10^{-4}$ and 10 , which leads to $0.7>\sigma / A$ $>10^{-3}$. The typical grid sizes that were used are 1024 $\times 1024,1024 \times 2048$, and $1024 \times 512$. The mesh size $d$ has been adjusted to get a sufficient numerical precision of the temperature solution in the case of a parallel plate configuration where an analytical solution is known. The numerical stability of the solutions has also been tested against slight shifts of the mesh position on an oversampled self-affine aperture field, $2 n_{x} \times 2 n_{y}=2^{12} \times 2^{12}$, and against the introduction of a lower cutoff $l_{c}$ of the self-affine perturbations varying between the mesh size and ten times the mesh size: the determined flow and temperature fields were found to be independent of such small-scale modifications.

\section{HYDRAULIC AND THERMAL FLOW EQUATIONS}

\section{A. Hydraulic flow}

We consider the steady flow of a Newtonian fluid at low Reynolds number, so that the viscous term of the NavierStokes equation dominates the inertial one. The NavierStokes equation is therefore reduced to the Stokes equation $[51,52]$

$$
\nabla P=\eta \Delta \boldsymbol{v},
$$

where $\eta$ is the dynamic viscosity, $\boldsymbol{v}$ is the velocity of the fluid, and $P$ is the pressure deviation from the hydrostatic profile (i.e., the hydraulic head which is equal to the pressure corrected by the gravity effect). To be in the framework of the lubrication approximation [37], besides small Reynolds number, we also consider fractures with flat enough sides as

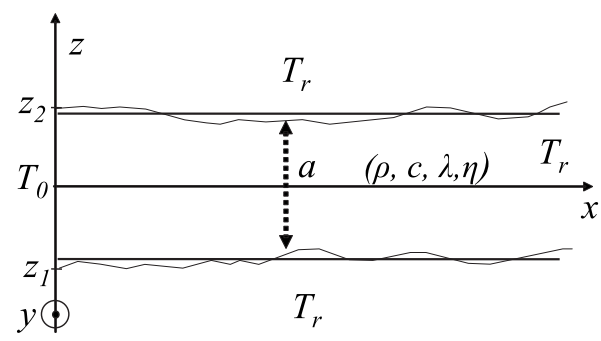

FIG. 1. 2D sketch of the fracture model with parameter definitions. $x$ axis is along the mean hydraulic flow, $y$ axis is along the mean fracture plane but perpendicular to the main hydraulic flow, and $z$ axis denotes the out-of-mean fracture plane direction. $z=z_{1}$ and $z=z_{2}$ are the average positions of the facing fracture surfaces. $a(x, y)$ is the fracture aperture. $T_{r}$ is the temperature of the solid, supposed to be homogeneous and constant, $T_{0}$ is the fluid temperature at the inlet. Fluid properties are $\rho, c, \chi$, and $\eta$, which are, respectively, density, heat capacity, thermal diffusivity, and dynamic viscosity.

mentioned above (i.e., with small local slopes). Therefore, fluid velocity vectors get negligible $z$ components (normal to the mean fracture plane), and accordingly the velocity field is dominated by in-plane components. The unitary vector $\hat{\boldsymbol{x}}$ is aligned with the macroscopic imposed pressure gradient (see Fig. 1$) ; z_{1}(x, y)$ and $z_{2}(x, y)$ are the bottom and top fracture coordinates, with $z_{2}-z_{1}=a$. Under these approximations, the pressure dependence is $P(x, y)$ and the velocity $\boldsymbol{v}$ is oriented along the unitary vector $\hat{\boldsymbol{v}}(x, y)$. By integrating the Stokes equation with the boundary conditions, $\boldsymbol{v}\left(z_{1,2}\right)=\mathbf{0}$, we get a local parabolic law in $z$ (Fig. 2) [25]:

$$
\boldsymbol{v}(x, y, z)=\frac{\boldsymbol{\nabla}_{\mathbf{2}} P(x, y)}{12 \eta}\left(z-z_{1}\right)\left(z-z_{2}\right),
$$

where $\boldsymbol{\nabla}_{\mathbf{2}}=\hat{\boldsymbol{x}} \frac{\partial}{\partial x}+\hat{\boldsymbol{y}} \frac{\partial}{\partial y}$ is the in-plane gradient operator.

Integrating Eq. (4) along $z$ leads to expressing the hydraulic flow through the fracture thickness $\boldsymbol{q}$ as

$$
q=-\frac{a^{3}}{12 \eta} \nabla_{2} P
$$

Furthermore, we assume the fluid to be incompressible, i.e., $\boldsymbol{\nabla}_{\mathbf{2}} \cdot \boldsymbol{q}=0$, which leads to the Reynolds equation [19]

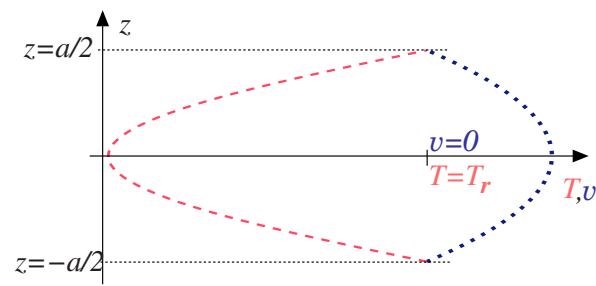

FIG. 2. (Color online) Local velocity quadratic profile (short dashed line) and temperature quartic profile (long dashed line) inside a fracture [with coefficients from Eqs. (9) and (4)]; arbitrary abscissa units. Along the contact with the fracture, $\boldsymbol{v}=\mathbf{0}$ and $T=T_{r}$. 


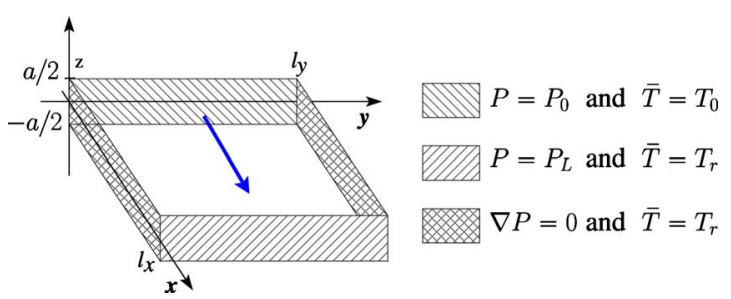

FIG. 3. (Color online) Fracture model with pressure and temperature boundary conditions.

$$
\boldsymbol{\nabla}_{\mathbf{2}} \cdot\left(a^{3} \boldsymbol{\nabla}_{\mathbf{2}} P\right)=0 .
$$

As boundary conditions of this equation (Fig. 3), we impose the pressure at the inlet and outlet of the fracture (if $x=0$, $P=P_{0}$, and if $x=l_{x}, P=P_{L}$, with $\left.P_{0}>P_{L}\right)$ and consider impermeable sides (no mass exchange with the rock matrix) at $y=0$ and $y=l_{y}$.

\section{B. Thermal flow}

In this work, we neglect the natural convection that happens in fractured rocks when the fluid density is thermally sensitive, as studied, for instance, by Bataillé et al. [10]. Natural convection might happen within the thickness of the fracture (owing to the temperature difference between the fracture boundary and the core of the flow along the gravity direction) and at large scale when the fracture is nonhorizontal. For the sake of simplicity, we consider that the forced fluid flow studied here is only weakly affected by density changes. A quantitative criterion of this assumption is given by the comparison of the pressure differences $\Delta P$ forcing the flow and that generated by the temperature changes: $\Delta P$ $\gg g \alpha_{T} \rho \Delta T$, with $g$ as the gravity, $\alpha_{T}$ as the fluid coefficient of thermal expansion, $\rho$ as the fluid density, and $\Delta T$ as the temperature differences in the system. We also assume that the Prandtl number of the fluid is sufficiently high for the flow to be dominated by hydrodynamic effects rather than thermal effects.

Since our focus is to understand how the macroscopic mass and heat flows are affected by the fracture roughness in the stationary limit, we do not consider time and space variations of the temperature in the rock: the fracture sides are assumed to be permanently hot at the fixed temperature $T_{r}$. This simplification is valid if we consider either long time scales, i.e., when the rock temperature profiles stabilizes, or time scales shorter than that of the hosting rock evolution. Taking the slow temperature evolution of the hosting rock into account would require us to combine the present study with a nonstationary conductive heat solver for the rock, which is beyond the scope of this paper. In principle, to model these intermediate time scales, the macroscopic parameters controlling the heat exchange determined in this paper could be utilized in a hybrid model, coupling the heat diffusion-advection in the fluid with the heat diffusion in the solid.

Local energy conservation implies that the fluid temperature is controlled by the balance between thermal convection and conduction inside the fluid, which reads as (with heat source due to friction between fluid layers being neglected) [53]

$$
\boldsymbol{v} \cdot \boldsymbol{\nabla} T=\chi \Delta T,
$$

where $\chi$ is the thermal diffusivity of the fluid and $T$ is the fluid temperature. We extend the lubrication approximation (cf. Sec. I) by considering that the slopes of the fracture morphology are small enough to provide conduction at the rock interface locally oriented along $\hat{z}$. This implies that the out-of-plane conduction term is dominant in front of the inplane ones. Otherwise, $v_{z} \partial T / \partial z$ can be neglected in $\boldsymbol{v} \cdot \boldsymbol{\nabla} T$ since out-of-plane velocity $v_{z}$ is negligible. Accordingly the leading terms in Eq. (7) are the conduction along the $\hat{z}$ axis and the in-plane convection terms, and this reduces to

$$
\frac{\partial^{2} T}{\partial z^{2}}=\frac{v_{x}}{\chi} \frac{\partial T}{\partial x}+\frac{v_{y}}{\chi} \frac{\partial T}{\partial y} .
$$

For the boundary conditions, we assume that the fluid temperature is equal to the rock temperature along the fracture sides,

$$
T\left(x, y, z_{1,2}\right)=T_{r},
$$

and far from the fracture inlet,

$$
T(x, y, z) \underset{x \rightarrow \infty}{\rightarrow} T_{r}
$$

The temperature of injection at the inlet is $T_{0}$, so that $T(0, y, z)=T_{0}$ (for any $y$ and $z$ ). By assuming that $\beta$ $=q_{x} \partial T / \partial x+q_{y} \partial T / \partial y$ is only a function of $x$ and $y$, the following quartic expression of $T$ is a solution of Eq. (8):

$$
T(x, y, z)=T_{r}-\frac{\beta(x, y)}{2 a^{3} \chi}\left(z-z_{1}\right)\left(z-z_{2}\right)\left(z-\sqrt{5} z_{1}\right)\left(z-\sqrt{5} z_{2}\right) \text {. }
$$

For the particular case of symmetric apertures around an average plane, i.e., where $z_{1}=-z_{2}=a / 2$, this reduces to $T$ $=-3 \beta\left(z^{4} / 6-a^{2} z^{2} / 4+5 a^{4} / 96\right) /\left(a^{3} \chi\right)+T_{r}$. By uniqueness of the solution for given boundary conditions (the problem is well posed), this quartic law is the only solution of Eq. (7). The temperature profile along $z$ is illustrated together with the velocity profile in Fig. 2 .

The energy conservation equation [Eq. (7)] is integrated along the $z$ direction through the thickness of the fracture (as done for the hydraulic description), which provides an inplane description of the thermal balance. First, we estimate the advected energy flux. For this, we note by $c$ the fluid specific heat capacity and $U_{0}$ its internal energy density at $T=T_{0}$, and write the internal energy density $U$ as $U=U_{0}$ $+\rho c\left(T-T_{0}\right)$. Integrating along the fracture thickness (i.e., along the $z$ axis) leads to the internal energy flux per unit volume $\boldsymbol{f}(x, y)=\int U \boldsymbol{v} d z$, which can be expressed as

$$
\boldsymbol{f}(x, y)=\left[U_{0}+\rho c\left(\bar{T}-T_{0}\right)\right] \boldsymbol{q}(x, y),
$$

where $\bar{T}$ is a weighted average temperature defined as 


$$
\bar{T}(x, y)=\frac{\int_{a} v(x, y, z) T(x, y, z) d z}{\int_{a} v(x, y, z) d z},
$$

with $\bar{T}(0, y)=\bar{T}_{0}=T_{0}$ at the inlet. The heat source coming from the advected energy is then given by $-\nabla_{2} \cdot f$. Using the mass conservation equation, $\boldsymbol{\nabla}_{\mathbf{2}} \cdot \boldsymbol{q}=0$, leads to

$$
\nabla_{2} \cdot f=\rho c q \cdot \nabla_{2} \bar{T} .
$$

The advected energy flux balances the conductive flux through the upper and lower fracture walls. To evaluate the thermal conductive flow oriented along the outgoing normal to the fracture walls $\hat{\boldsymbol{n}}$, the lubrication approximation (cf. Sec. I), leads to $\hat{\boldsymbol{n}} \simeq \pm \hat{\boldsymbol{z}}$. Let $\varphi_{w}$ be the projection of the conductive flow along $\hat{\boldsymbol{n}}$, evaluated along the walls, at $z_{1,2}$. The Fourier law provides $\varphi_{w}=-\left.\chi \rho c \frac{\partial T}{\partial z}\right|_{z=z_{12}} \hat{z} \cdot \hat{\boldsymbol{n}}$. Inserting Eqs. (9) and (4) to Eq. (11) leads to $\left.\frac{\partial T}{\partial z}\right|_{z=z_{1,2}}=\left(\bar{T}-T_{r}\right) \frac{70}{17 a} \hat{z} \cdot \hat{\boldsymbol{n}}$. The Nusselt number $\mathrm{Nu}=-\varphi_{w} / \varphi_{\text {ref }}=70 / 17$ is used to characterize the efficiency of the present heat exchange compared to the reference heat flow $\varphi_{r e f}=\chi \rho c\left(T_{r}-\bar{T}\right) / a$, which occurs in situations with only conduction. The energy net flux,

$$
\nabla_{2} \cdot f+2 \varphi_{w}=0
$$

can finally be expressed as

$$
\boldsymbol{q} \cdot \nabla_{\mathbf{2}} \bar{T}+2 \frac{\chi}{a} \mathrm{Nu}\left(\bar{T}-T_{r}\right)=0 .
$$

For the boundary conditions of the two-dimensional field $\bar{T}$, we assume that the fluid is injected at a constant temperature $\bar{T}(0, y)=T_{0}$ colder than the rock, and we consider the length of the fracture to be long enough to get the fluid at the same temperature as the rock at the end of it: $\bar{T}\left(l_{x}, y\right)=T_{r}$. On the contrary, temperature settings along the boundaries $y=0$ and $y=l_{y}$ have no influence since the hydraulic flow is null there [see Eq. (6)].

Let the reference case be a fracture modeled with two parallel plates separated by a constant aperture $a_{\|}$(i.e., no self-affine perturbation). In this case, the gradient of pressure is constant all along the fracture, as well as the hydraulic flow, which is equal to $\boldsymbol{q}_{\|}=-\Delta P a_{\|}^{3} /\left(12 l_{x} \eta\right) \hat{\boldsymbol{x}}$, where the subscript $\|$ denotes results valid for parallel plates and $\Delta P=P_{L}$ $-P_{0}$. Under these conditions Eq. (14) is invariant along $y$ and can be written as

$$
\frac{\partial \bar{T}_{\|}}{\partial x}+\frac{\left(\bar{T}_{\|}-T_{r}\right)}{R_{\|}}=0,
$$

where the thermal length $R_{\|}$characterizes the distance at which the fluid reaches the temperature of the surrounding rock:

$$
R_{\|}=\frac{a_{\|}\left\|\boldsymbol{q}_{\|}\right\|}{2 \mathrm{Nu}_{\|} \chi}=-\frac{\Delta P}{l_{x}} \frac{a_{\|}^{4}}{24 \eta \mathrm{Nu}_{\|} \chi},
$$

with $\mathrm{Nu}_{\|}=70 / 17 \simeq 4.12$. Then the analytical solution of Eq. (15) for parallel plates is

$$
\bar{T}_{\|}-T_{r}=\left(T_{0}-T_{r}\right) \exp \left(-\frac{x}{R_{\|}}\right) .
$$

For rough fractures, we aim at using Eq. (17) as a proxy of the average temperature profile $\overline{\bar{T}}$ along the flow and defining an effective macroscopic thermal length $R$ as

$$
\overline{\bar{T}}-T_{r}=\left(T_{0}-T_{r}\right) \exp \left(-\frac{x}{R}\right) .
$$

\section{Definition of microscopic and macroscopic apertures}

Different types of fracture apertures can be defined. The most obvious one is the geometrical aperture, but effective apertures such as hydraulic or thermal aperture can also be introduced. The latter are defined on the basis of an inversion on a specific model like the parallel plate model. For instance, the hydraulic aperture is deduced from the knowledge of the fluid flow through the fracture, and it represents the aperture of a parallel plate model that reproduces the observed fluid flow. Equivalently a thermal aperture can be introduced as the aperture of a parallel plate model that reproduces a similar thermal profile. A spatial scale has to attach to define the hydraulic or thermal equivalent behavior, in particular for a multiscale geometry. Since we aim at understanding the upscaling of the fracture properties, we will introduce two specific scales: the smallest one, i.e., the grid size of the discretization, and the largest one, i.e., the system size. The smallest will be referred to as the microscopic or local scale and small letters will be used for their labeling, and the largest will be referred to as the macroscopic scale and described with capital letters.

We already use the microscopic geometrical or mechanical aperture $a$ and its spatial average, i.e., the macroscopic geometrical aperture: $A=\langle a(x, y)\rangle_{x, y}$. The microscopic hydraulic aperture is defined as from Eq. (5) $[19,54]$

$$
h=\left(\|\boldsymbol{q}\| \frac{12 \eta}{\frac{\Delta P}{l_{x}}}\right)^{1 / 3} .
$$

It depends on the local hydraulic flow $\boldsymbol{q}$ and can be related to the local pressure gradient $\|\nabla P\|$ and local aperture $a$ as

$$
h=a\left(\frac{\|\boldsymbol{\nabla} P\|}{\frac{\Delta P}{l_{x}}}\right)^{1 / 3} .
$$

If the local pressure gradient $\|\nabla P\|$ is smaller than the macroscopic gradient $\Delta P / l_{x}$, then $h(x, y)<a(x, y)$, which means that locally the hydraulic conductivity is lower than expected from its local mechanical aperture. The macroscopic hydraulic aperture $H$ can also be defined at the system scale from the average hydraulic flow $Q_{x}=\left\langle\boldsymbol{q} \cdot \boldsymbol{n}_{x}\right\rangle_{x, y}$ : 


$$
H=\left(Q_{x} \frac{12 \eta}{\left|\frac{\Delta P}{l_{x}}\right|}\right)^{1 / 3}
$$

Macroscopic and microscopic hydraulic apertures are related since $H$ is actually proportional to the cubic root of the thirdorder moment of $h: H=\left\langle h(x, y)^{3}\right\rangle_{x, y}^{1 / 3}$, which is proportional to the first-order moment of the hydraulic flux to power of $1 / 3$. If $H / A>1$, then the fracture is more permeable than parallel plates separated by $a(x, y)=A$.

The macroscopic thermal aperture is defined from a onedimensional temperature profile $\overline{\bar{T}}(x)$ along the forced pressure gradient direction [see Eq. (18)] where the average temperature is defined as

$$
\overline{\bar{T}}(x)=\frac{\int_{l_{y}} u_{x}(x, y) \bar{T}(x, y) d y}{\int_{l_{y}} u_{x}(x, y) d y} .
$$

It is an average of $\bar{T}$ along the width of the fracture $l_{y}$, weighted by the local fluid velocity $u_{x}(x, y)$ $=q_{x}(x, y) / a(x, y)$ which is the ratio of the $x$ component of the local flux over the local fracture aperture. Then, by fitting the parallel plate temperature solution [Eq. (18)] to the average temperature profile $\overline{\bar{T}}(x)$, we get the macroscopic thermal length $R$. In practice the fit is computed from a least-squares minimization, for abscissa from $x=0$ to the minimum $x$ value, so that $\left|\left(\overline{\bar{T}}-T_{r}\right) /\left(T_{0}-T_{r}\right)\right|<2 \times 10^{-6}$. The macroscopic thermal aperture $\Gamma$ is then defined by analogy to the parallel plate solution [Eq. (16)] as

$$
\Gamma=\left(-R \times 24 \eta \mathrm{Nu} \chi \frac{l_{x}}{\Delta P}\right)^{1 / 4}=A\left(R^{*}\right)^{1 / 4},
$$

where $R^{*}=R / R_{\|}$is the normalized thermal length. At a coarse-grained scale, the rough fracture is thermally equivalent to parallel plates separated by the constant aperture $a(x, y)=\Gamma$. Indeed, both will exhibit the same thermal length $R$ under the same macroscopic pressure gradient $\Delta P / l_{x}$.

The microscopic thermal aperture $\gamma$ can also be introduced after defining a local thermal length $r$. Similarly to the definition of a microscopic hydraulic aperture from the local pressure gradient, or local flux, rather than the macroscopic pressure difference, or macroscopic flux, we estimate the local thermal length from a local temperature gradient rather than a large-scale pressure difference. Equation (14) can be rewritten as

$$
\boldsymbol{q} \cdot \nabla_{\mathbf{2}}\left(\ln \left[\bar{T}^{*}\right]\right)+\frac{\|\boldsymbol{q}\|}{r}=0
$$

with

$$
r=\frac{a\|q\|}{2 \mathrm{Nu} \chi},
$$

which is an estimate of the gradient along $\hat{\boldsymbol{s}}$, the local hydraulic flow direction. Finally, the local thermal aperture $\gamma$ can be defined by [consistently with Eq. (16)]

$$
\gamma=\left(-r \times 24 \eta \mathrm{Nu} \chi \frac{l_{x}}{\Delta P}\right)^{1 / 4} \text {. }
$$

A link between macroscopic and microscopic thermal apertures can also be shown as follows: at first order, considering that the average of $\|\boldsymbol{q}\|$ is very close to the average of $q_{x}$ then the local length of reference would be equal to $\tilde{r}=-\left[\partial \ln \left(\bar{T}^{*}\right) / \partial x\right]^{-1}$. On the other hand, integrating Eq. (18) between zero and significant length $L$ results in $R=-\left(\left\{\ln \left[\bar{T}^{*}(L)\right]-\ln \left[\bar{T}^{*}(0)\right]\right\} / L\right)^{-1}$, which shows the link between macroscopic and microscopic thermal apertures: $R=L\left[\int_{0}^{L}(-1 / \widetilde{r}) d x\right]^{-1}$, i.e., $R=\left\langle r^{-1}\right\rangle^{-1} \propto\left\langle(a\|\boldsymbol{q}\|)^{-1}\right\rangle^{-1}$, according to Eq. (25).

For parallel plates, all microscopic apertures are equal and also equal to the macroscopic ones: $h=a=\gamma=H=\Gamma=A$. For rough fractures, this is definitively not the case since the local apertures vary spatially inside the fracture. We will see in the next section how all these apertures are influenced by the roughness amplitude of the fracture aperture, for which we will emphasize on two main parameters: the normalized root-mean-square deviation $\sigma / A$ of the geometrical aperture and the aspect ratio of the fracture $J=l_{x} / l_{y}$, i.e., the ratio of the downstream length of the fracture $l_{x}$ over its width $l_{y}$.

\section{Dimensionless quantities}

Dimensionless positions, apertures, pressure, temperature, and hydraulic flow are defined as follows:

$$
\begin{gathered}
x^{*}=\frac{x}{d}, \quad y^{*}=\frac{y}{d}, \\
a^{*}=\frac{a}{A}, \quad H^{*}=\frac{H}{A}, \quad \Gamma^{*}=\frac{\Gamma}{A}, \\
P^{*}=-\frac{\left(P-P_{0}\right)}{2 d} \frac{l_{x}}{\Delta P}, \\
\bar{T}^{*}=\frac{\bar{T}-T_{r}}{T_{0}-T_{r}}, \\
\boldsymbol{q}^{*}=-\frac{12 \eta l_{x}}{\Delta P A^{3}} \boldsymbol{q},
\end{gathered}
$$

where $d$ is the mesh size of the aperture grid. Otherwise, we note that in the dimensionless temperature, the difference between the injection temperature $T_{0}$ and the rock temperature $T_{r}$ intervenes only as a factor of proportionality.

\section{HYDRAULIC FLOW SIMULATIONS}

\section{A. Description of the pressure solver}

The Reynolds and temperature equations [Eqs. (6) and (14)] are numerically solved by using a finite difference scheme. The pressure $P$, the hydraulic flow $\boldsymbol{q}$, and tempera- 
ture $\bar{T}$ are discretized on a grid of $n_{x} \times n_{y}$ points with a mesh size of $2 d$ i.e., half of the aperture grid points. In the following, when indices $(i, j)$ are positive integers, they refer to node positions where an aperture, a pressure, and a temperature are defined on the contrary to the noninteger node position $(i \pm 0.5$ or $j \pm 0.5)$ where only an aperture is defined.

The Reynolds equation [Eq. (6)] is discretized and solved in the same way as by Méheust and Schmittbuhl [20]: we use finite differences centered on a square mesh of lattice step size $2 d$, and the linear equation system is inverted using an iterative biconjugate gradient method [49]. The chosen pressure drop along the fracture length is $\Delta P^{*}=P_{n_{x}, j}^{*}-P_{1, j}^{*}=1$ $-n_{x}$ for $1 \leq j \leq n_{y}$. The hydraulic flow $\boldsymbol{q}_{i, j}^{*}=\left(q_{i, j x}^{*}, q_{i, j y}^{*}, 0\right)$ is computed from the pressure field as

$$
\begin{aligned}
& q_{i, j x}^{*}=-\frac{a_{i, j}^{*^{3}}}{2}\left(P_{i+1, j}^{*}-P_{i-1, j}^{*}\right), \\
& q_{i, j y}^{*}=-\frac{a_{i, j}^{*^{3}}}{2}\left(P_{i, j+1}^{*}-P_{i, j-1}^{*}\right) .
\end{aligned}
$$

For a parallel plate configuration (i.e., modeling without selfaffine perturbation), $q_{i, j_{x}}^{*}=1$ and $q_{i, j_{y}}^{*}=0$ everywhere in the fracture.

\section{B. Example of a microscopic hydraulic aperture field}

An example of a fracture aperture is shown in Fig. 4(a). It is generated as explained in Sec. II on a $1024 \times 512$ grid and has a RMS equal to $\sigma / A=0.25$. The hydraulic flow computed inside this morphology is shown in Fig. 4(b), as well as the microscopic hydraulic apertures [Fig. 4(c)]. In this case, the hydraulic flow exhibits a strong channeling as previously described by Méheust and Schmittbuhl [20]. The microscopic hydraulic apertures can be observed not to be simply correlated with the aperture field.

The link between microscopic mechanical apertures $a$ and the microscopic hydraulic apertures $h$ is given in Fig. 5, where the scale shows the corresponding occurrence probability of each local configuration. We see that the normalized mechanical and hydraulic aperture values are distributed around a characteristic point: $(h / A,\langle a\rangle / A)=(1,1)$. Nevertheless, the correlation between both apertures is not simple. Some of the highest density values are located below and above the straight line which represents $h=a$. Accordingly, the permeability can locally be lower or higher than what is given by an average Poiseuille law. The scattering around the straight line shows that, at one point, the local flow is not determined by the local mechanical aperture, but is influenced by all the surrounding microscopic mechanical apertures. From computations with other $\sigma$ 's, we notice that the lower the roughness amplitude is, the closer to $(1,1)$ the cloud is.

\section{Variability of the macroscopic hydraulic aperture}

The dimensionless macroscopic hydraulic aperture is measured for our fracture example as $H / A=0.94$ (ordinate of the cross in Fig. 5). $H / A<1$ means that the fracture perme-
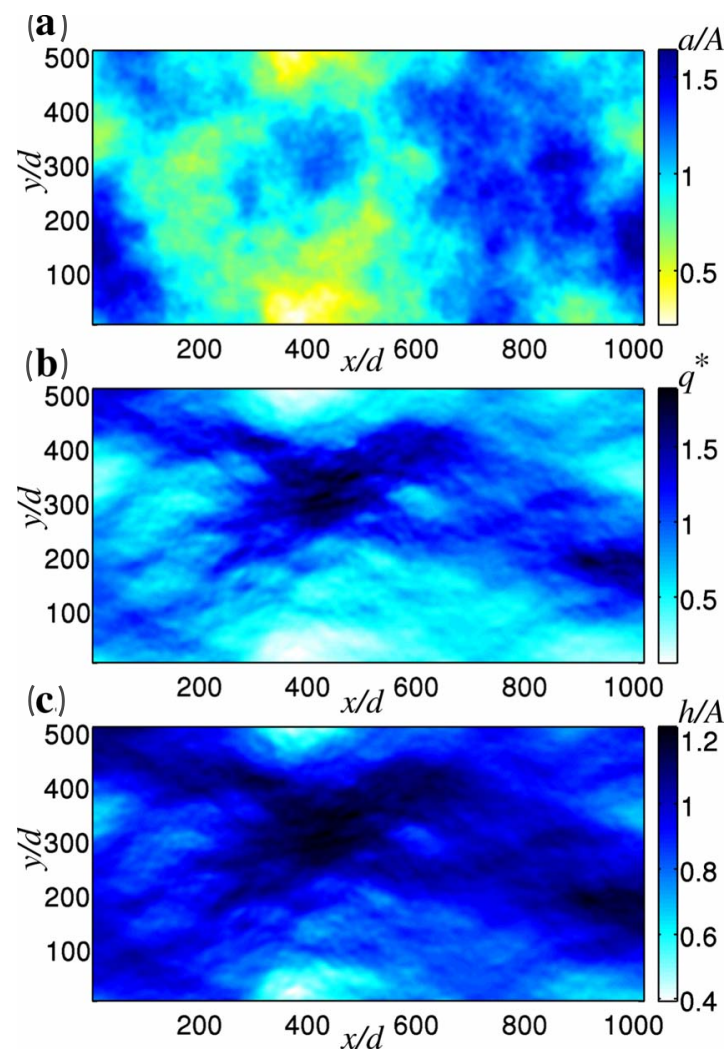

FIG. 4. (Color online) (a) Self-affine aperture with $\sigma / A=0.25$. (b) Dimensionless hydraulic flow norm computed with the aperture of (a), having for dimensionless hydraulic aperture $H^{*}=0.94$. (c) Microscopic hydraulic apertures, computed from the third root of the hydraulic flow shown in (b).

ability is reduced compared to the one of parallel plates having the same mechanical aperture $A$, without any self-affine perturbation. For the same morphology pattern (Fig. 4), we examine how the roughness amplitude influences the macroscopic hydraulic aperture by changing $\sigma / A$ (cf. Sec. II). In Fig. 6 we see that the macroscopic hydraulic aperture is close to 1 when $\sigma / A=0.05$, which corresponds to a quasiflat aperture. When the roughness amplitude increases, $H$ decreases,

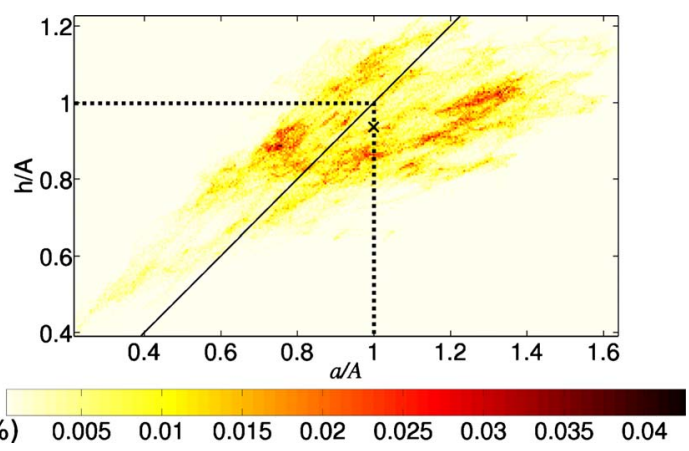

FIG. 5. (Color online) 2D histogram of the link between the microscopic hydraulic aperture and the microscopic mechanical aperture for the fracture shown in Fig. 4 (the scale indicates the probability in percent); the cross has for coordinates $(H / A,\langle a\rangle / A)$ $=(0.94,1)$. The straight line is $h=a$, which is the equality given by a local Poiseuille law. 


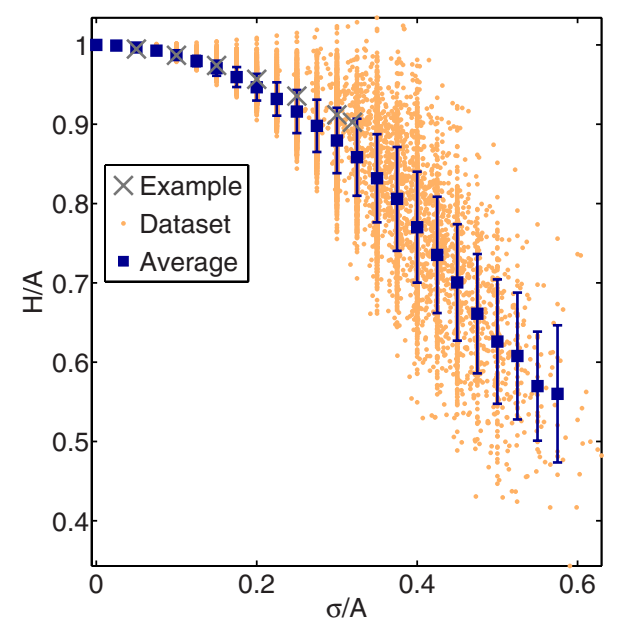

FIG. 6. (Color online) Macroscopic hydraulic aperture $H / A$ versus $\sigma / A$ for fractures with aspect ratio $l_{x} / l_{y}=2$; crosses, variation of the hydraulic aperture by increasing the roughness amplitude $\sigma / A$ for the aperture shown in Fig. 4; dots, cloud of computed data (about 20000 aperture realizations); squares, average hydraulic behavior with variability bars. On average, $H / A<1$ : the permeability is smaller than expected from the Poiseuille law in parallel plate apertures.

which means that this morphology pattern tends to inhibit the hydraulic flow and makes the fracture permeability decrease.

For various realizations with the same $\sigma / A$ value, various hydraulic behaviors may happen owing to the channel variability in the hydraulic flow. In Fig. 6, we plot the dimensionless macroscopic hydraulic apertures $H / A$ versus $\sigma / A$ (for about 20000 computations with 1700 different fracture aperture patterns). Here, each fracture has the same size as the fracture shown in Fig. 4 where $l_{x} / l_{y}=2$. We compute the mean hydraulic apertures inside windows of size $0.025 \sigma / A$, and each plotted bar represents twice the standard deviation of $H / A$ inside the corresponding windows. We see that, for most cases, the permeability is reduced. For $\sigma / A<0.25$, the hydraulic aperture is still quite close to $A$, and the dispersivity is relatively small even if some configurations show a flow enhancement owing to the fracture roughness: $H>A$ [20]. Then, for higher RMS, the average of $H / A$ decreases significantly on average (up to $50 \%$ ) with $\sigma / A$, but with a higher variability of the results.

\section{Influence of the fracture aspect ratio on the hydraulic flow}

To get a complete description, we now modify one additional parameter: the aspect ratio of the fracture, by changing the ratio of the fracture length over its width, $J=l_{x} / l_{y}$. Figure 7 shows the same kind of average plots of $H / A$ as a function of $\sigma / A$ but for three different aspect ratios: $J=2$ (square symbols), which is the one presented in Fig. $6, J=1$ (triangle), and $J=0.5$ (disks). Since less simulations were done for $J=1$ and $J=2$ (see the legend in Fig. 7), few apertures show $\sigma / A>0.45$, and therefore no average points are represented in these cases. For square systems $(J=1)$ and downstream elongated fracture $(J \geq 1), H / A$ is on average smaller than 1 (i.e., inhibiting hydraulic flow compared to the one

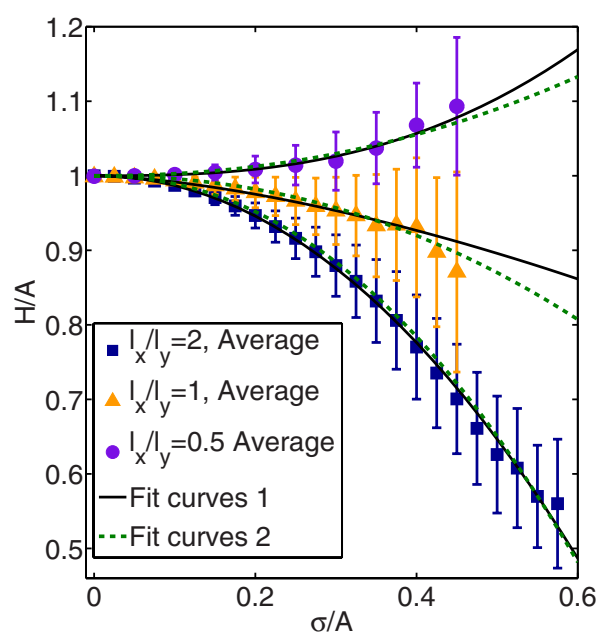

FIG. 7. (Color online) Macroscopic hydraulic aperture versus $\sigma / A$, for three aspect ratios $J=l_{x} / l_{y}$. Averages computed from data are shown with symbols, with error bars, corresponding to plus or minus the standard deviation (see how the average is computed in Sec. IV C). $J=l_{x} / l_{y}=2$ shows an enhanced flow (same data as presented in Fig. 6); $J=1$ shows on average a slightly inhibited flow, i.e., $H \leq A$ (computed from a cloud of about 1300 points); for $J$ $=0.5$, on average, higher permeability is observed (computed from a cloud of about 900 points). Continuous curves are fitting models (1) $H / A=1+\alpha\left(\frac{\sigma}{A}\right)^{\kappa}$, with parameters $(\kappa, \alpha)$ equal to $(2.05,-1.46)$, $(1.57,-0.31),(2.69,0.67)$, respectively, for $J$ equal to 2,1 , and 0.5 . Dotted curves are obtained with fitting models (2) $H / A=1$ $-\mu\left[\log _{2}(J)+\delta\right]\left(\frac{\sigma}{A}\right)^{\kappa}$, with $(\mu, \delta, \kappa)=(0.98,0.59,2.16)$, for the three curves.

through parallel plates separated by the same opening $A$ ), whereas for systems wider perpendicularly to the pressure gradient direction, $H / A$ is on average higher than 1. A qualitative explanation might be that it is statically more likely to get a large-scale connecting channel for a wide and short fracture $(J<1)$ rather than for a thin and long fracture $(J$ $>1)$. In other words, qualitatively, channels are rather in parallel in wide fractures and in series in long ones. For square systems which should be isotropic and providing as many perpendicular and parallel channels, we see that when the roughness amplitude increases, the hydraulic aperture gets on average slightly smaller than $A$. We can suspect that there would exist an aspect ratio $J_{i n v}$, so that the hydraulic aperture is on average independent of the fracture roughness magnitude: $H / A=1$ for any $\sigma / A$. Following the model proposed below in Sec. IV E, we get $J_{i n v} \simeq 0.65 \pm 0.05$. For any $J$ value, we see that the higher the ratio $\sigma / A$ is, the higher the variability of the behaviors is, especially for square systems which exhibit both high $(H>A)$ and low $(H<A)$ permeabilities for the same roughness magnitude.

\section{E. Model of the average macroscopic hydraulic aperture}

One of the main questions we want to address here is the relationship between the macroscopic hydraulic aperture $H$ and the microscopic mechanical aperture field $a(x, y)$. The knowledge of the mechanical aperture field $a(x, y)$ provides us the following bounds for $H:\left\langle a^{-3}\right\rangle^{-1}<H^{3}<\left\langle a^{3}\right\rangle$ - the 
lower case corresponds to a system of aperture fluctuations purely aligned in series, and the upper one corresponds to fluctuations purely aligned in parallel [55]. However, $a(x, y)$ is rarely known and subsequently $\left\langle a^{-3}\right\rangle^{-1}$ and $\left\langle a^{3}\right\rangle$ are difficult to estimate.

From Fig. 7, $\sigma / A$ and $J$ appear to be important parameters controlling the macroscopic hydraulic aperture of the fracture $H$. Reference [20] proposed a first model of the $H$ behavior as $H / A=1+\alpha\left(\frac{\sigma}{A}\right)^{\kappa}$. Here, we similarly model the average hydraulic aperture curves corresponding to each aspect ratio (continuous curves in Fig. 7) and find $(\kappa, \alpha)$ successively equal to $(2.05,-1.46),(1.57,-0.31)$, and $(2.69,0.67)$, respectively, for $J$ equal to 2,1 , and 0.5 . Depending on the sign of $\alpha$, we get a permeability either lower or higher than that expected with flat plates. Then we fit these three behaviors by a more general model which includes the aspect ratio variation, with three parameters $(\mu, \delta, \kappa)$ to be optimized: $H / A=1-\mu\left[\log _{2}(J)+\delta\right]\left(\frac{\sigma}{A}\right)^{\kappa} . \quad$ With $\quad(\mu, \delta, \kappa)=(0.98,0.59$, $2.16)$, we get the three dotted lines in Fig. 7 which are acceptable fits of the average trend. However, it has to be highlighted that the real hydraulic aperture of a specific surface is possibly very different from this average value (see the size of variability bars in Fig. 7), especially at high $\sigma / A$.

Other models for numerical or experimental hydraulic apertures have been proposed in the literature [19] as $(H / A)^{3}$ $=1-C_{1} \exp \left(-C_{2} A / \sigma\right)$ or $(H / A)^{3}=1 /\left[1+C_{3}(2 A / \sigma)^{1.5}\right]$, where $C_{1-3}$ are constants, but the shape of these functions does not fit well our averaged points, and these fits are not presented here.

\section{THERMAL FLOW SIMULATIONS}

\section{A. Description of the temperature solver}

The temperature equation [Eq. (14)] is discretized as

$$
q_{i, j_{x}}^{*}\left(\bar{T}_{i+1, j}^{*}-\bar{T}_{i-1, j}^{*}\right)+q_{i, j y}^{*}\left(\bar{T}_{i, j+1}^{*}-\bar{T}_{i, j-1}^{*}\right)+\frac{4 d}{R_{\|}} \frac{\bar{T}_{i, j}^{*}}{a_{i, j}^{*}}=0,
$$

where $(i, j) \in\left[\left|2, n_{x}-1\right|\right] \times\left[\left|2, n_{y}-1\right|\right]$ and $R_{\|}$is the thermal length expected by neglecting the roughness amplitude [see Eq. (16)]. The boundary conditions are

$$
\begin{gathered}
1 \leq j \leq n_{y}, \quad \bar{T}_{1, j}^{*}=1, \quad \bar{T}_{n_{x}, j}^{*}=0, \\
2 \leq i \leq n_{x}-1, \quad \bar{T}_{n_{x}, j}^{*}=0, \quad \bar{T}_{n_{x}, j}^{*}=0 .
\end{gathered}
$$

The system is solved in the same way as the pressure system (Sec. IV A). Two limiting numerical factors intervene for the efficiency of the discretization scheme: the mesh step $d$ has to be sufficiently small to capture with a sufficient accuracy the relative variations of $\bar{T}-T_{r}$ over a lattice step. In practice, the mesh step used in this paper is chosen as $d$ $=R_{\|} / 50$. We checked that dividing this mesh size by 2 did not change significantly the computed temperature field. The second numerical limit is that the system size $l_{x}$ has to be larger than $20 R_{\|}$to avoid a possible numerical instability (mostly with the aperture grid size $1024 \times 2048$, which is

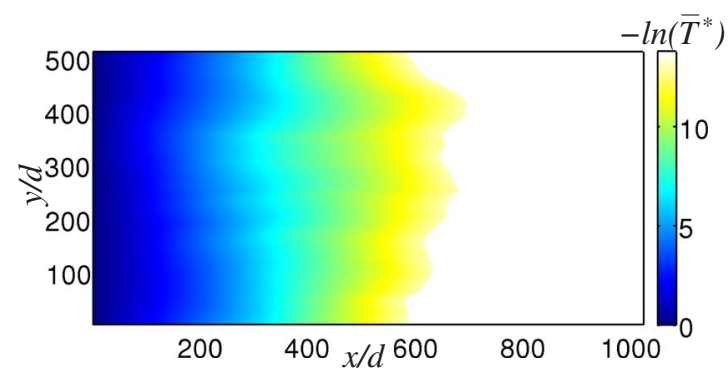

FIG. 8. (Color online) $-\ln \left(\bar{T}^{*}\right)$, opposite of the logarithm of the temperature field $\bar{T}^{*}$ computed from the aperture morphology pattern shown in Fig. 4 with a very low roughness amplitude: $\sigma / A$ $=0.05$. The hydraulic aperture of this fracture is $H / A=0.99$. The temperature field exhibits a normalized thermal length equal to $R^{*}$ $=0.97$ and a thermal aperture of $\Gamma / A=0.99$.

more likely to exhibit a longer thermal length, as explained in Sec. V E). If not, the fluid passing the fracture is so slowly warmed up that the condition $\bar{T}_{n_{x}, j}^{*}=0$ at the outlet badly represents the condition imposed in principle at infinity in the channel, and this boundary condition imposed at a physically too short distance from the inlet cannot be fulfilled without numerical artifact. To face this problem in such rare situations, we duplicate the aperture grid to get a longer system length and impose the same macroscopic pressure gradient, and the rock temperature at the new end: $\bar{T}_{2 \cdot n_{x}, j}^{*}=0$.

\section{B. Example of a local microscopic temperature field}

For a nearly constant aperture $(\sigma / A=0.05)$, we numerically obtain a temperature law close to an exponential downstream profile (Fig. 8), as we expect from Eq. (17). The 2D temperature field shown in Fig. 9(a) $(\sigma / A=0.25)$ is computed from the aperture and its previously computed hydrau-

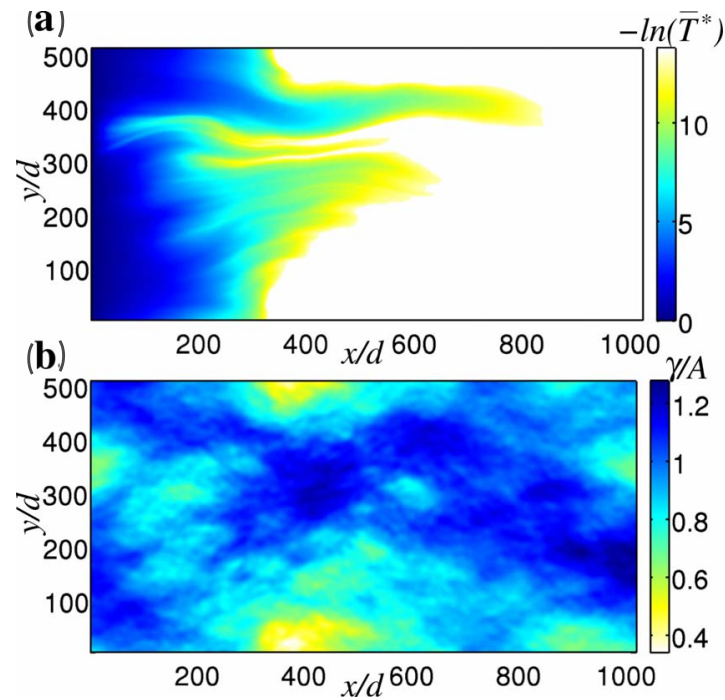

FIG. 9. (Color online) (a) $-\ln \left(\bar{T}^{*}\right)$, opposite of the logarithm of the 2D temperature field, computed from the apertures in Fig. 4(a) $(\sigma / A=0.25)$. (b) Normalized local thermal aperture $\gamma / A$ associated with the temperature field shown in (a). 


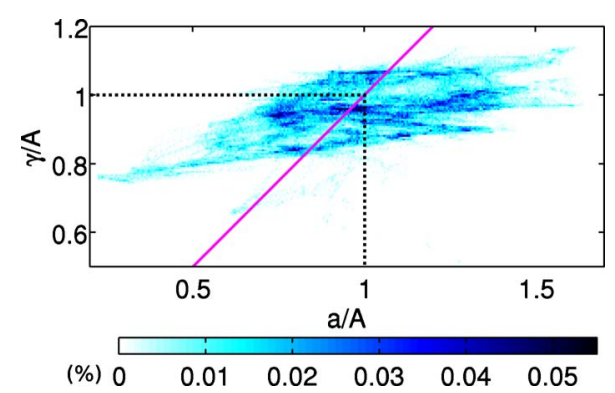

FIG. 10. (Color online) 2D histogram in percent of the fracture shown in Fig. 4 as a function of the local thermal aperture $\gamma$ and local aperture $a$ (the shading indicates the probability density). The straight line is $\gamma=a$. The dispersivity of the cloud around the line shows that there is no simple link between the local aperture and the thermal one.

lic flow field, shown in Fig. 4(b). We see that it can be observed that the fluid is getting inhomogeneously warm, with channelized features. The thermal channel begins in a zone where the hydraulic flow coming from the inlet converges [Fig. 4(b)]. The local normalized thermal aperture $\gamma / A$ [map shown in Fig. 9(b)] exhibits less pronounced channel effect than in Fig. 9(a). Figure 10 is the plot of the local microscopic thermal apertures $\gamma / A$ versus the local apertures $a / A$, using a shading showing the occurrence density in the $(\gamma / A, a / A)$ space. The dispersivity of the cloud around the line $\gamma=a$ shows that there is no simple link between the local aperture and the thermal one. A similar plot (Fig. 11) allows us to observe the correlation between the local microscopic thermal apertures and the local microscopic hydraulic apertures. It shows a good correlation of the local thermal aperture and the local hydraulic aperture (i.e., the cloud is close to the straight line $\gamma=h)$. Note that it is more probable $(59 \%)$ to have $\gamma>h$, which corresponds to a heat exchange locally less efficient than what is expected from a parallel plate model which is equivalent in permeability.

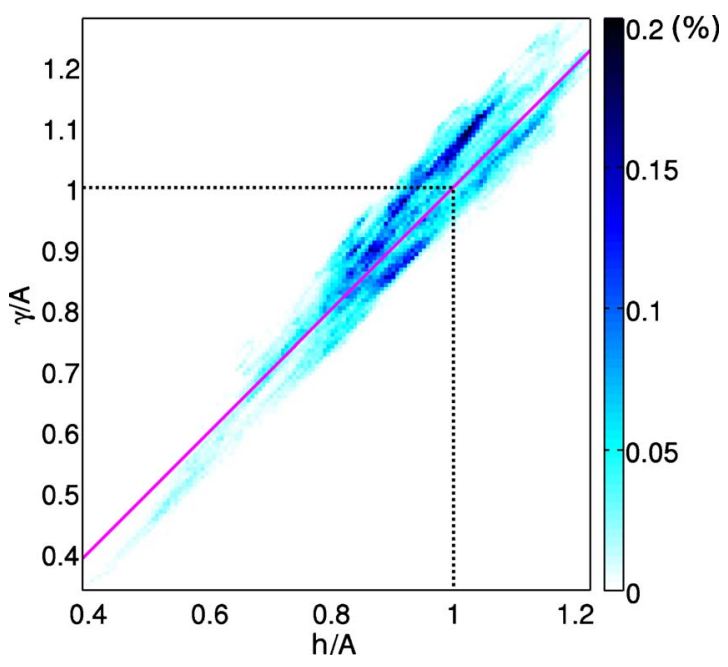

FIG. 11. (Color online) 2D Histogram in percent of the fracture shown in Fig. 4 as a function of the local thermal aperture $\gamma$ and local hydraulic aperture $h$ (the scale indicates the probability in percent). The straight line is $\gamma=h$; the localization of the cloud around the line shows a good correlation between $\gamma$ and $h$.

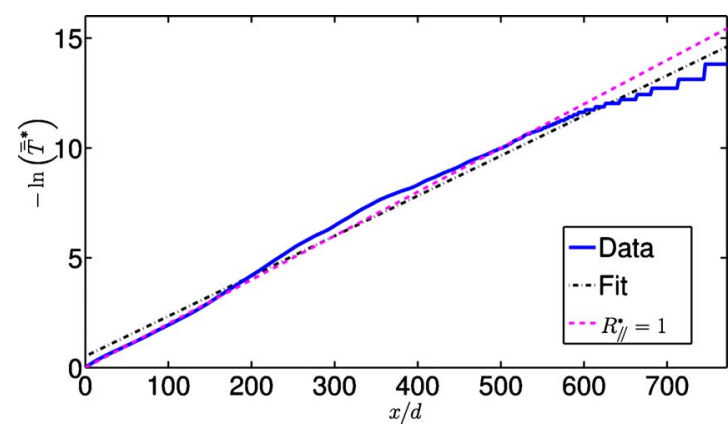

FIG. 12. (Color online) Continuous curve, $-\ln \left(\overline{\bar{T}}^{*}\right)$, opposite of the logarithm of the temperature field computed from the temperature field $\bar{T}$ shown in Fig. 9. Dashed-dotted curve, linear fit of curve A (from $x / d=0$ to $x / d=772$ ), which provides the thermal length:

$-\ln \left(\overline{\bar{T}}^{*}\right)=x / 1.09+0.6$, i.e., $R^{*}=1.09$. Dashed curve, $-\ln \left(\overline{\bar{T}}_{\|}^{*}\right)$, opposite of the logarithm of the temperature law for the same fracture modeled without self-affinity perturbation (i.e., parallel plates), which has for thermal length $R_{\|}^{*}=1$.

\section{Variability of the macroscopic thermal aperture}

The average temperature $\overline{\bar{T}}$ [see definition in Eq. (22)] is a semilocal property which shows how the thermal behavior evolves on average along the pressure gradient direction. The shape of $\overline{\bar{T}}(x)$ (Fig. 12) is close to an exponential law, but with a thermal length $R$ slightly different from the fracture without self-affine perturbation (i.e., parallel plates). This thermal length is computed from the slope of the linear regression of $\ln [\overline{\bar{T}}(x)]$ (see Sec. III C). In the example displayed in Fig. 12, the thermal length is $R_{\|}^{*}=1.09$, which results in an equivalent thermal aperture of $\Gamma^{*}=1.02$.

In Fig. 13, the crosses illustrate the roughness amplitude influence on the thermal aperture for the morphology pattern

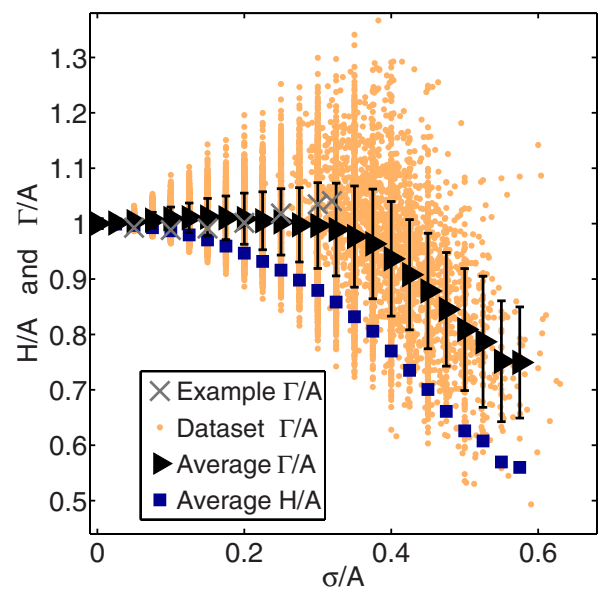

FIG. 13. (Color online) Crosses, variation of the thermal aperture $\Gamma / A$ by increasing the roughness amplitude $\sigma / A$ for the aperture pattern shown in Fig. 4; dots, cloud of computed data (about 20000 points) for fractures with aspect ratio $l_{x} / l_{y}=2$; triangles, average thermal behavior with variability bars of the cloud; squares, average hydraulic aperture $H / A$ versus $\sigma / A$, recalled here for comparison. 


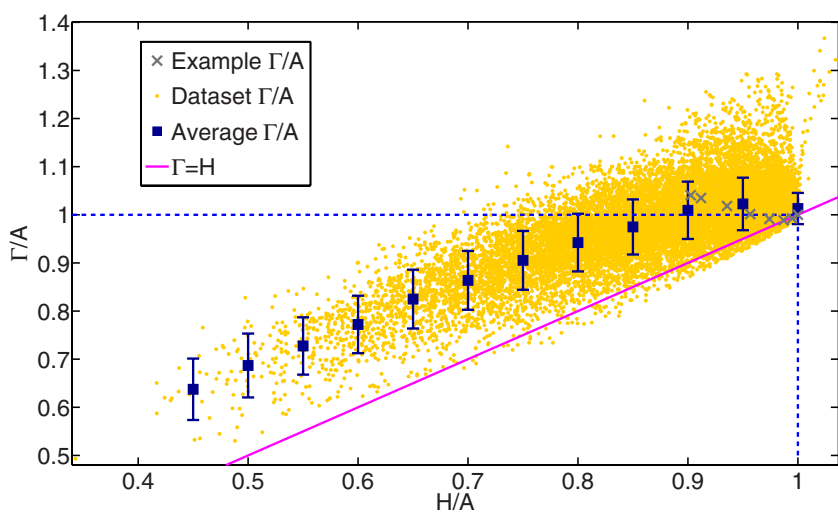

FIG. 14. (Color online) Normalized thermal aperture $\Gamma / A$ versus $H / A$ for fractures with aspect ratio $l_{x} / l_{y}=2$. Crosses, variation of the thermal aperture by increasing the roughness amplitude for the aperture pattern shown in Fig. 4(a) versus $H / A$; dots, cloud of computed data (about 20000 points); squares, average thermal behavior with variability bars. Continuous curve, $\Gamma / A=H / A$, which holds for parallel plates separated by $a(x, y)=H$.

shown in Fig. 4(a), whose relief is amplified by changing the $\sigma$ value (see Sec. II). For this example, $\Gamma$ vs $\sigma$ is not monotonic. The dimensionless thermal length is close to 1 when $\sigma / A=0.05$, which corresponds to a quasiflat aperture. When the roughness amplitude is big enough $(\sigma>0.1), \Gamma$ increases with $\sigma$ and is higher than 1 , which means that this morphology pattern tends to inhibit the thermal exchange. In Fig. 14, the crosses show the thermal aperture versus $H / A$ using the same data as for the plots shown by crosses in Figs. 6 and 13.

\section{Variability of the thermal behavior}

Statistical thermal results are computed for numerous cases (more than 20000 ) whose macroscopic hydraulic apertures are presented in Sec. IV C for various $\sigma / A$ values. Similarly, a normalized average macroscopic thermal aperture, $\Gamma / A$, and its standard deviation is obtained as functions of $\sigma / A$. The resulting $\Gamma / A$ for the aspect ratio $J=2$ is displayed in Fig. 13, with bars representing the double of the standard deviation. For the same normalized roughness amplitude $\sigma / A$, various thermal behaviors may happen, especially for $\sigma / A>0.25$, with channels appearing or not and dimensionless thermal lengths higher or lower than 1. At first order, both the macroscopic thermal (Fig. 13, triangles) and hydraulic average apertures (Fig. 13, squares) are decreasing as functions of $\sigma$. This trend is significantly more pronounced for $H$ than for $\Gamma$. The thermal results are compared with systems equivalent in permeability (same normalized hydraulic aperture) in Fig. 14 which presents the normalized thermal aperture versus the hydraulic aperture with the average points computed inside windows of size $0.05 H / A$. The most striking result is that roughness inhibits thermalization: nearly all the cloud is above the continuous curve $\Gamma=H$, which means that the thermalization of the fluid (thermalization is obtained when the fluid temperature reaches the rock one) is inhibited compared to what we expect from the hydraulic behavior. At the same time, we note that, on average, $\Gamma / A<1$, i.e., most of the apertures exhibit an enhanced ther-

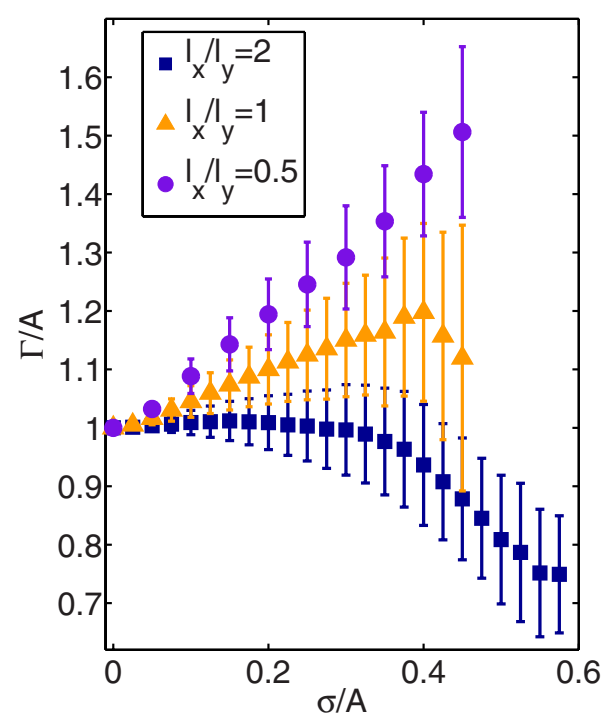

FIG. 15. (Color online) Averages of the normalized thermal aperture $\Gamma / A$ and their deviation bars versus $\sigma / A$ for various aspect ratios $J=l_{x} / l_{y}$, as indicated by the labels. See how the average is computed in Sec. IV C.

malization compared to what would be expected with a model of flat fractures separated by $A$, i.e., having the same geometric (or mechanical) aperture.

\section{E. Influence of the fracture aspect ratio on the thermal behavior}

We complete our study by computing the averaged thermal apertures for two other aspect ratios, $J$, by using the hydraulic flows computed in Sec. IV D. The averaged values of the thermal apertures, with the variability bars (defined similarly to what is done in Sec. V D) for $J \in\{0.5,1,2\}$ are plotted in Figs. 15 and 16. When $\Gamma / A$ is plotted as a function of $\sigma / A$ (Fig. 15), various thermal behaviors are observed, according to the aspect ratio, with high variability, particularly when $\sigma / A>0.25$. On the contrary to fractures with aspect ratio equal to $J=2$ (described in Sec. V D) the ones with $J$ in $\{0.5,1\}$ are more likely to inhibit the thermalization compared to flat fractures with the same mechanical aperture $(\Gamma / A>1)$.

Figure 16 shows the average of $\Gamma / A$ versus $H / A$. Contrarily to what can be observed for $\Gamma / A$ vs $\sigma / A$ (Fig. 15), the average curves $\Gamma / A$ vs $H / A$ are roughly independent on the aspect ratio. This shows that the hydraulic aperture is a better parameter than the roughness $\sigma / A$ to assess the thermal properties. The thermal aperture is systematically larger than the hydraulic aperture $(\Gamma>H)$. It means that once the permeability is known, e.g., by pumping tests, using a parallel plate model separated by $H$ for estimating the thermal behavior overestimates the efficiency of the heat exchange: the fluid needs indeed a longer distance to be thermalized than expected from flat fractures with the same permeability. On average $\Gamma / A$ vs $H / A$ is monotonic (Fig. 16), i.e., this average dependence displays a simpler behavior than for a particular case of morphology of varying amplitude (e.g., Fig. 14, crosses). 


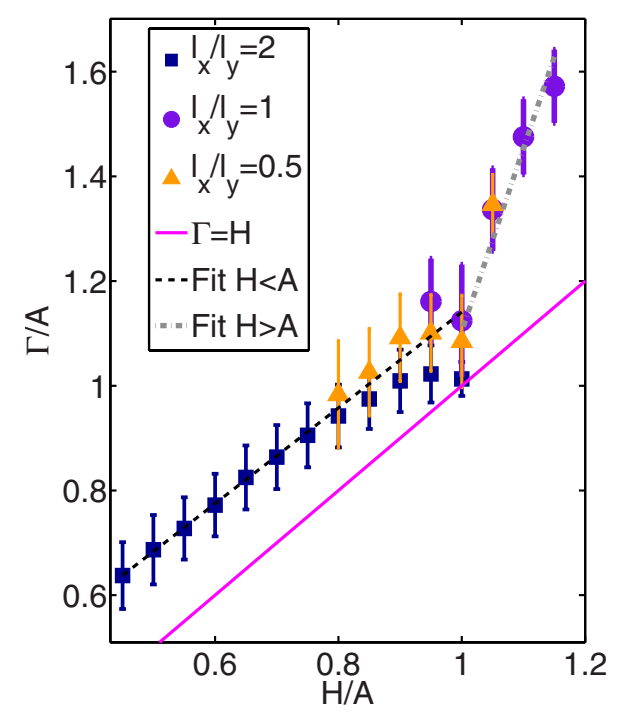

FIG. 16. (Color online) Averages of the normalized thermal aperture $\Gamma / A$ and their deviation bars versus $H / A$ for various aspect ratios $J=l_{x} / l_{y}$, as indicated by the labels (see how the average is computed in Sec. IV C). Models lines are $\Gamma=0.9 H+0.2 A$ for $H$ $<A$ and $\Gamma=3.5 H-2.4 A$ for $H \geq A$; no continuity condition between both lines is imposed.

Going more into details, Fig. 16 also shows that for $H / A>1$, the slope of $\Gamma$ vs $H$ is steeper than for $H / A<1$; both parts of the curve can be modeled with straight line fits (dotted and dotted-dashed curves). This could be interpreted as follows: fractures with high hydraulic apertures provide high velocities, so that fluid particles need to go further to reach the rock temperature. Fractures with small hydraulic apertures $H / A<1$ might be dominated by small mechanical apertures (fences) providing small velocities, which leads to thermal apertures closer to the line $\Gamma=H$.

\section{DISCUSSION AND CONCLUSION}

\section{A. Model limits and possible extension}

Despite the hydraulic lubrication hypothesis, which implies notably a low Reynolds number, the fluid velocity should not be too small. Indeed, the velocity drives the inplane thermal convection, which is supposed to be large compared to the in-plane thermal conduction. This can be quantified by the Péclet number (ratio between the characteristic times of diffusion and advection): our model is valid at low in-plane Péclet number. Therefore, owing to in-plane conduction, the thermal channeling effect might be reduced especially in the case of high temperature contrast along the channel and very low hydraulic flow. This homogenization might be reinforced if the fluid temperature is still inhomogeneous but very close to the rock temperature: in this case the in-plane conduction inside the fluid might be as high as the conduction between rock and fluid. Free convection (temperature dependence of $\rho$ ), which is not taken into account here, may also intervene, especially for thick fractures [56].

In practice, some $3 \mathrm{D}$ effects might happen as the lubrication approximation is not necessarily respected owing to the rock morphology (e.g., $[23,24])$. In natural cases, the roughness amplitude $\sigma / A$ covers a large range across the natural cases, from small to large values according to the type of rock and fractures. For instance, we recently measured the roughness amplitude of natural fractures in black marl at borehole scale, and we obtained values of $\sigma / A<0.04$ for one and $\sigma / A=0.3$ for another one [22]. Some other values, typically $\sigma / A>0.4$, have also been reported, for instance, in granitic rocks $[57,58]$. If the cases with large roughness amplitudes also correspond to large local slopes (angle between the fracture side and the average plane), it is likely that the Reynolds equation and 2D temperature equation do not apply so well to these cases, and that the results reported here are only approximate for those.

When the fracture morphology is highly developed, due to more surface exchange, the rock might locally provide better heat exchange. The assumption of averaging thermal phenomena in two dimensions has been studied, e.g., by Volik et al. [59] and Sangaré et al. [60], who considered only conduction. The 3D solving of the full Navier-Stokes and heat advection-diffusion equations is also possible, for example, with a coupled lattice-Boltzmann method [61]. However, considering the complexity of fracture morphology from very small scales to large ones requires heavy computations, which makes statistical results difficult to obtain. When convection also acts, 3D effects lead to zones decoupled from the main mass and heat flux, as the fluid might be blocked into eddies (off lubrication regime) provoked by sharp morphologies [23,29-32] (like Moffatt eddies [62]). It has indeed to be noticed that even when low-pressure gradient is imposed, turbulent flow might be observed due to high roughness amplitude. This effect is complementary to observations made at high Reynolds number [63-66], when even a very low roughness amplitude of the wall induces turbulent flow.

All the results about the thermal aperture may also be influenced by the thermal boundary conditions. In particular we have assumed that $T_{r}$ is constant. Spatial variations of $T_{r}$ can easily be taken into account by changing the boundary conditions of the thermal equation while temporal variations require to model the rock getting colder in the surrounding (consequences of the rock diffusivity). In time, the hypothesis of constant temperature $T_{r}$ holds either for very short durations when the regime is transitory or for longer durations, at quasistationary regime, when the rock temperature evolves very slowly and the fluid temperature adapts fast. This is the case if the solid is much more thermally diffusive than the fluid, which is quite true in our case: for instance, the ratio of the granite thermal diffusivity over the water one is 5.9 . We could check the time evolution by using another numerical approach based on lattice-Boltzmann methods [61], which allows us to solve both the rock and fluid temperatures and takes into account the contrast of thermal diffusivity. For a fracture with an aperture of a few millimeters, $T_{r}$ can be considered as constant at transitory regime for durations much less than $1 \mathrm{~min}$. Conversely it also holds for longer durations after a quasistationary regime is reached, which can happen after minutes or years, according to the properties of the system (e.g., distance to the heat source and injection point). Time variation of $T_{r}$ can also be taken into 
account by coupling our model to a thermal diffusion model in the rock, using alternately both models in time. Similarly, it is possible to couple our code to another one modeling the change in the geometry of the fracture (e.g., because of stress or chemical reactions).

\section{B. Conclusion}

We have proposed a model of thermal exchange between a Newtonian fluid and a hot rock inside a rough fracture under a given pressure gradient. The flow considered was assumed to be at low Reynolds number, in laminar regime, so that Stokes equation and lubrication approximations hold for the mass flow equations and for the temperature advection in the heat transport equation. We have then set from basic principles the mass and heat transport equations, expressed them in a $2 \mathrm{D}$ form, discretized them by finite differences, and solved the resulting systems by biconjugate gradient methods. The whole numerical scheme can be used with any variable aperture field without contact (for instance, obtained from real rock surfaces). Here, apertures have been chosen to be numerically generated, in order to get statistically significant results over more than 20000 realizations. The aperture fields are modeled as many natural ones, namely, as self-affine with a Hurst exponent of 0.8 , with various ratios of the aperture fluctuations over the average aperture and three different aspect ratios of the fracture. The hydraulic and thermal behaviors are quantified with both local and macroscopic apertures: $h, \gamma$, and $H, \Gamma$.

The plot of $H$ as function of $\sigma / A$ exhibits some trends according to the aspect ratio, and we have been able to find model curves. However, around these model curves, the hydraulic behavior is very variable and, therefore, knowing the roughness amplitude, $\sigma / A$, these model curves may not be reliably combined with a model of another phenomenon dominated by the hydraulic aperture, such as the thermal exchange. The macroscopic thermal aperture $\Gamma$ vs the roughness amplitude is also highly variable, despite trends that are visible on average according to the aspect ratio. The fracture, taking into account its roughness, is either less or more permeable than model of flat parallel plates with the same mechanical aperture.

At local and macroscopic scales, hydraulic apertures are badly correlated with mechanical apertures. On the contrary, hydraulic apertures are highly correlated with thermal apertures, showing that the thermal behavior is mostly determined by the hydraulic one for rough fractures. Compared to flat fractures with equivalent permeability, for a rough aperture, the fluid almost systematically needs a longer distance to reach the temperature of rock $(\Gamma>H)$ : the heat exchange is less efficient. A practical implication of this general result is that when fracture aperture is assessed on the field based on hydraulic transmissivity measurement, obtained, e.g., by pumping tests, the efficiency of the thermal exchange assessed from flat fracture models is systematically overestimated. Using the laws $\Gamma=0.9 H+0.2 A$ if $H<A$ and $\Gamma$ $=3.5 H-2.4 A$ for $H \geq A$ (Fig. 16) should allow us to correct this overestimation.

Another important result is that the derived temperature in rough channels, when averaged, behaves according to the solution of the macroscopic equation that would be used for flat apertures [Eq. (14)]. The local roughness of the fracture can therefore be coarse grained. Doing so, the structure of Eq. (14) is kept; it is simply necessary to adjust both the hydraulic transmissivity and the thermal length (or the Nusselt number). For instance, this coarse-grained approach based on parallel plate laws with adjusted Nusselt numbers can be used for hydraulic and thermal models of fracture networks.

\section{ACKNOWLEDGMENTS}

We thank E. G. Flekkøy, S. Roux, K. J. Måløy, J. Andrade, Jr., H. Auradou, A. Genter, and J. Sauze for fruitful discussions. We thank the European EHDRA program, as well as the ANR ECOU-PREF and the REALIZE programs for their financial support.
[1] A. Gérard, A. Genter, T. Kohl, P. Lutz, P. Rose, and F. Rummel, Geothermics 35, 473 (2006).

[2] A. Neuville, R. Toussaint, and J. Schmittbuhl, Proceedings of the EHDRA Scientific Conference, 2006 (unpublished).

[3] A. Neuville, R. Toussaint, and J. Schmittbuhl, C. R. Geosci. 342, 616 (2010).

[4] J. Sausse, C. Dezayes, A. Genter, and A. Bisset, Proceedings of the 33rd workshop on Geothermal Reservoir Engineering, Stanford University, CA, 2008 (unpublished).

[5] T. Le Borgne, O. Bour, J. Dreuzy, P. Davy, and F. Touchard, Water Resour. Res. 40, W03512 (2004).

[6] V. Cvetkovic, S. Painter, N. Outters, and J. Selroos, Water Resour. Res. 40, W02404 (2004).

[7] A. Jupe et al., Geothermics 24, 403 (1995).

[8] J.-R. De Dreuzy, C. Darcel, P. Davy, and O. Bour, Water Resour. Res. 40, W01502 (2004).

[9] P. Adler and J.-F. Thovert, Fractures and Fracture Networks
(Kluwer Academic, Dordrecht, 1999).

[10] A. Bataillé, P. Genthon, M. Rabinowicz, and B. Fritz, Geothermics 35, 654 (2006).

[11] E. Bouchaud, J. Phys.: Condens. Matter 9, 4319 (1997).

[12] L. Ponson, H. Auradou, M. Pessel, V. Lazarus, and J.-P. Hulin, Phys. Rev. E 76, 036108 (2007).

[13] Y. Méheust and J. Schmittbuhl, Pure Appl. Geophys. 160, 1023 (2003).

[14] D. Bonamy, L. Ponson, S. Prades, E. Bouchaud, and C. Guillot, Phys. Rev. Lett. 97, 135504 (2006).

[15] F. Plouraboué, P. Kurowski, J.-P. Hulin, S. Roux, and J. Schmittbuhl, Phys. Rev. E 51, 1675 (1995).

[16] A. Neuville, R. Toussaint, J. Schmittbuhl, D. Koehn, and J. Schwarz, special issue of Hydrolog. Process. (to be published).

[17] Y. Méheust and J. Schmittbuhl, Geophys. Res. Lett. 27, 2989 (2000).

[18] F. Plouraboué, P. Kurowski, J.-M. Boffa, J.-P. Hulin, and S. 
Roux, J. Contam. Hydrol. 46, 295 (2000).

[19] S. Brown, J. Geophys. Res. 92, 1337 (1987).

[20] Y. Méheust and J. Schmittbuhl, J. Geophys. Res. 106, 2089 (2001).

[21] J. Schmittbuhl, A. Steyer, L. Jouniaux, and R. Toussaint, Int. J. Rock Mech. Min. Sci. 45, 422 (2008).

[22] A. Neuville, R. Toussaint, and J. Schmittbuhl, special issue of Hydrolog. Process. (to be published).

[23] S. Brown, H. Stockman, and S. Reeves, Geophys. Res. Lett. 22, 2537 (1995).

[24] V. Mourzenko, J.-F. Thovert, and P. Adler, J. Phys. II 5, 465 (1995).

[25] R. Zimmerman and I.-W. Yeo, Geophysical Monograph (American Geophysical Union, Washington, D.C., 2000), Vol. 122 , p. 213.

[26] F. Plouraboué, J.-P. Hulin, S. Roux, and J. Koplik, Phys. Rev. E 58, 3334 (1998).

[27] R. Zimmerman and G. Bodvarsson, Transp. Porous Media 23, 1 (1996)

[28] G. Drazer and J. Koplik, Phys. Rev. E 66, 026303 (2002).

[29] D. Brush and N. Thomson, Water Resour. Res. 39, 1085 (2003).

[30] A. Al-Yaarubi, C. Pain, C. Grattoni, and R. Zimmerman, in Dynamics of Fluids and Transport in Fractured Rocks, edited by B. Faybishenko, P. Witherspoon, and J. Gale, AGU Monograph Vol. 162 (American Geophysical Union, Washington, D.C., 2005), pp. 55-64.

[31] D. Boutt, G. Grasselli, J. Fredrich, B. Cook, and J. Williams, Geophys. Res. Lett. 33, L21402 (2006).

[32] M. Cardenas, D. Slottke, R. Ketcham, and J. Sharp, Jr., Geophys. Res. Lett. 34, L14404 (2007).

[33] N. Heuer, T. Kupper, and D. Windelberg, Geophys. J. Int. 105, 659 (1991).

[34] S. Gentier, X. Rachez, C. Dezayes, A. Hosni, A. Blaisonneau, A. Genter, and D. Bruel, Proceedings of the EHDRA Scientific Conference, 2005 (unpublished).

[35] X. Rachez, S. Gentier, and A. Blaisonneau, Proceedings of the EHDRA Scientific Conference, 2007 (unpublished).

[36] O. Kolditz and C. Clauser, Geothermics 27, 1 (1998).

[37] O. Pinkus and B. Sternlicht, Theory of Hydrodynamic Lubrication (McGraw-Hill, New York, 1961).

[38] D. Turcotte and G. Schubert, Geodynamics, 2nd ed. (Cambridge University Press, Cambridge, England, 2002), Chap. 6, pp. 262-264.

[39] K. Falconer, Fractal Geometry: Mathematical Foundation and Application (Wiley, New York, 1990).
[40] J. Feder, Fractals (Plenum, New York, 1988).

[41] F. Plouraboué, S. Roux, J. Schmittbuhl, and J.-P. Vilotte, Fractals 51, 1675 (1995).

[42] J. Schmittbuhl, S. Gentier, and S. Roux, Geophys. Res. Lett. 20, 639 (1993).

[43] J. Schmittbuhl, F. Schmitt, and C. Scholz, J. Geophys. Res. 100, 5953 (1995).

[44] W. Power, T. Tullis, S. Brown, G. Boitnott, and C. Scholz, Geophys. Res. Lett. 14, 29 (1987).

[45] B. Cox and J. Wang, Fractals 1, 87 (1993).

[46] J. Boffa, C. Allain, and J.-P. Hulin, Eur. Phys. J.: Appl. Phys. 2, 281 (1998).

[47] Y. Méheust, Ph.D. thesis, Université Paris Sud, 2002.

[48] S. Roux, J. Schmittbuhl, J.-P. Vilotte, and A. Hansen, EPL 23, 277 (1993).

[49] W. Press, S. Teukolsky, W. Vetterling, and B. Flannery, Numerical Recipes (Cambridge University Press, New York, 1992).

[50] M. Sahimi, Phys. Rep. 306, 213 (1998).

[51] G. Stokes, Trans. Cambridge Philos. Soc. 8, 287 (1846).

[52] G. Batchelor, An Introduction to Fluid Dynamics (Cambridge University Press, New York, 1967).

[53] L. Landau and E. Lifchitz, Physique Théorique, Mécanique des Fluides, 3rd ed. (Ellipses, Moscow, 1994), Chap. 5, p. 280.

[54] R. W. Zimmerman, S. Kumar, and G. S. Bodvarsson, Int. J. Rock Mech. Min. Sci. Geomech. Abstr. 28, 325 (1991).

[55] M. J. Beran, Statistical Continuum Theories (Interscience, New York, 1968).

[56] H. Murphy, J. Geophys. Res. 84, 6121 (1979).

[57] E. Hakami and E. Larsson, Int. J. Rock Mech. Min. Sci. 33, 395 (1996)

[58] M. Vicente Silvestre, L. Ribeiro e Sousa, and R. Hack, News J., Int. Soc. Rock Mech. 7, 9 (2002).

[59] S. Volik, V. Mourzenko, J.-F. Thovert, and P. Adler, Transp. Porous Media 27, 305 (1997).

[60] D. Sangaré, J.-F. Thovert, and P. M. Adler, J. Appl. Phys. 104, 074915 (2008).

[61] A. Neuville, R. Toussaint, and J. Schmittbuhl (unpublished).

[62] H. Moffatt, J. Fluid Mech. 18, 1 (1964).

[63] J. Nikuradse, VDI-Forschungsh. 356, 1-35 (1932).

[64] G. Barenblatt, A. Chorin, and V. Prostokishin, Proc. Natl. Acad. Sci. U.S.A. 94, 773 (1997).

[65] E. Skjetne, A. Hansen, and J. Gudmundsson, J. Fluid Mech. 383, 1 (1999).

[66] P. Goldenfeld, Phys. Rev. Lett. 96, 044503 (2006). 